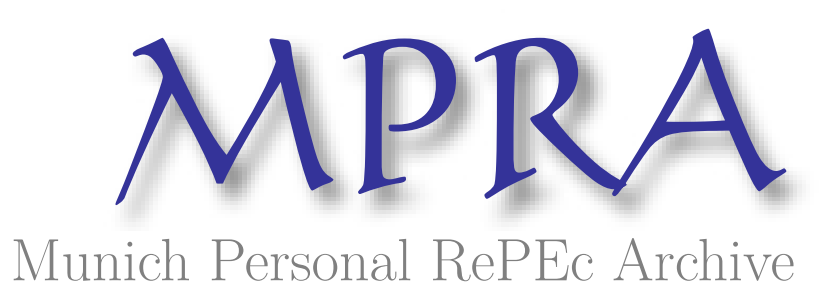

\title{
Behavior of Investors on a Multi-Asset Market
}

Steinbacher, Matjaz

June 2009

Online at https://mpra.ub.uni-muenchen.de/15898/

MPRA Paper No. 15898, posted 25 Jun 2009 00:02 UTC 


\title{
BEHAVIOR OF INVESTORS ON A MULTI- ASSET MARKET
}

\author{
Matjaz Steinbacher \\ Steinbacher.si \\ Financial analyst and advisor \\ Prvomajska ulica 7 \\ SI - 2310 Slovenska Bistrica, Slovenia \\ e-mail: matjaz.steinbacher@gmail.si \\ Phone: +38641960928
}

\begin{abstract}
This paper analyzes the field of investors' decision-making on a multi-asset market. It does it through a simulation games on a social network framework. It has been demonstrated that more stocks there are in the game and more changing alternatives investors have available to choose from, tougher it is for them to make decisions. Despite in most simulations the safest alternative was dominant, many investors opt for portfolio of the safest and the riskiest stock, by which they back the risk they take with some safe stocks. Non-omniscient investors behave chaotically. In all the cases, liquidity agents proved to be decisive elements of the games, though not always able to deliver the information of all the alternatives when too many alternatives are available.
\end{abstract}

Keywords: social networks, behavioral finance, portfolio analysis, multi-asset game, chaos.

JEL Classification: Z13, G11, C91, C73. 


\section{Introduction}

Simulations of a dynamic portfolio selection model with multiple assets are being done in the article. The article combines the theory of stochastic and behavioral finance, game theory, with the models of social networks, and continues my work on simulated portfolio games (Steinbacher 2008a, b; 2009a, b, c). In all previous games investors had to decide upon two separate assets and the combination of the two. This was quite easy task for them. However, managing portfolio is a much more complex game because many different kinds of assets make number of alternatives from which individual investors choose. I consider portfolio selection as such complex game in the article.

Perception of portfolio is the same as in Markowitz (1952) who puts it as a twostage process, with the first aiming at getting the knowledge of the prices of assets and the second of how to make a portfolio when the first stage is done. Such notion of a portfolio in fact reflects the existence of an uncertain future in financial markets, due to the stochastic nature of prices (Fama 1965; Campbell et al. 1997). Like Markowitz, I do not try to tackle the first stage, but focus only on the second. Daily and intraday movements on markets reflecting the behavior of selfish and goal-oriented individuals on markets would make such modeling superfluous. Besides, portfolio is the response to the stochastic nature of prices with individuals making decisions upon their beliefs and expectations (see Hirshleifer (2001) for a review on investor psychology in asset pricing). If prices were common knowledge, no one would take portfolio but the stock with the highest return. In fact, as long as prices are common knowledge, no trade is possible with no one be willing to sell below the common knowledge price and no one to buy above it. Therefore, I omit the first stage, take historical stock returns in time in a minute time intervals and simulate the decision-making of individuals in time upon the given returns.

Several pillars are significant in making simulations. When making decisions, investors have knowledge only of the current and past returns of alternatives they choose. They also possess the knowledge of the past returns that have been chosen by those to whom they are directly connected. Investors do not know the returns 
of alternatives they do not possess neither do they know the future returns of stocks they possess or alternatives they choose. Investors also do not know the mechanisms that drive the returns of assets in time. Therefore, investors decide upon their realized returns, from which they make expectations for the future. Since the future is not known with certainty, investors also consider uncertainty, as argued by Kahneman and Tversky (1979), and behave strategically. I also introduce such investors in the game that never change their initial strategy, whatever its efficiency. This is in line with the Rubinstein (1998), Osborne and Rubinstein (1990), and Kahneman and Tversky. The latter argue that investors have a strong desire to avoid the feeling of regret, by which some of them rather do nothing. Such assumption draws some very significant consequences for the decision-making of the entire group of investors, keeping the liquidity of temporary dominated strategies. Without such individuals, some consecutive poor outcomes of some alternatives could eliminate such alternatives from the scope of alternatives in the longer run. This process works as to the principle of an invisible hand. For this credits, I call them liquidity individuals (Steinbacher (2009b)).

Finally, investors in the game use a social network to communicate and share information with each other. Becker (1996) argues that individuals possess two sorts of capital: personal and social. Personal capital involves all of the previous experiences that influence or determine the current knowledge and behavior of an individual. Social capital determines all of the external effects of other individuals that contribute to the current and future level of knowledge of an individual. As such, personal capital and human capital represent only a part of an entire human capital of an individual.

In the network, each investor is connected to a small number of other investors, what represents individual's social capital. I assume that investors communicate with those to whom they are directly connected, by which they share information throughout the network. This is the most significant part of the simulation games, because investors get information they need to make decisions in time from their own experiences, as well as from the experiences of others. In an isolated world, investors would only depend on their own knowledge, which would limit the 
scope of the alternatives from which to choose. That would lead to superficial decisions. Individuals namely possess only a small bit of the entire knowledge (Hayek 1945), which makes the cooperation a requisite for the progress and better decisions. Levine and Pesendorfer (2007) argue that when individuals are involved in the social interaction, they are prone to modify their preferences and select the most efficient strategies out of the pool of strategies of their friends. The side effect of such cooperation within the network is herding, leading to synchronous decisions in time (Keynes 1936; Bikhchandani et al. 1998).

I assume that investors form a small world network, by which I assume that individuals have much stronger local contacts than global, but where individuals also have some of such global connections (Watts and Strogatz 1998; Wasserman and Faust 1994). For the efficient use of a social network, it is also important for individuals how capable they are in receiving information from others, how good the information they get from others is, how efficient they are to incorporate information into their knowledge and how good they use this in their decisionmaking. Another important aspect that affects the stock of individuals' knowledge is how prone agents are in sharing the information and knowledge they have with others. Rubinstein (1998) also brings forward some of the causes for the existence of constraints on the information held by an agent. The acquisition of information is costly; the information acquired often has to be stored in the memory before its use, and the memory is not unbounded; while information are received through the process of interaction that also has some of its own limits. If other agents are not prone of sharing their knowledge to others, such interaction does not spread the knowledge around the network. I do not consider the techniques by which investors communicate with each other, but assume that the communication among investors is perfect and efficient. This means that the two investors communicating with each other fully share their knowledge to one another.

The paper proceeds as follows. In the Section 2, I consider the social network used in the simulations, whereas in the Section 3, I propose a model of decisionmaking. In Section 4, I present the simulation results and the final Section 5 concludes. 


\section{Social network}

Agents are central feature of an agent-based approach, because they make decisions in time and share their knowledge. Russel and Norvig (1995) define agents as anything that is able to perceive the environment one is in by using sensors, and react upon the information one gets. In the model, I don not use machines or robots to make decisions, but software agents in a dynamic environment, which is changing in time. When modeling them, it is important what sort of knowledge they have, what are the ways they acquire new information, how they learn in time, how they process information they get into knowledge, how autonomous they are in their decision-making, and similar.

In a social network world, agents are involved in the social interaction with others and acquire new information by using communication skills with other agents to whom they are connected. This means that individuals are not isolated entities but as social beings who have relatives, friends, colleagues and other acquaintances with whom they communicate and share information. Interaction as such could be seen as a consequence of the fact that knowledge of an individual represents only a small portion of the entire knowledge, as argued by Hayek (1945).

A social network $G=(V, E)$ describes relations between individuals and is composed of finite the set of nodes (or vertices) $V$, representing individuals, and finite set of edges (or links) $E$, representing their pairwise relations. By using a adjacency matrix, a network is defined as $N \times N$ matrix $\mathbf{A}$, with $\mathbf{A}_{i, j}=1$ if $(i, j) \in E$ and 0 otherwise.

In the simulation games, I employ an undirected network. In an undirected network, edges are unordered pairs of nodes. That is, if $\mathbf{A}_{i, j}=1 \Leftrightarrow \mathbf{A}_{j, i}=1$ and if $\mathbf{A}_{i, j}=0 \Leftrightarrow \mathbf{A}_{j, i}=0$. In a directed network, edges have directions. The network being used here is a complete network, which means that all pairs of nodes in the network are connected and none isolated. 
The next significant characteristic of a network is the node degree $k$. Loosely speaking, it reflects to the number of connections an individual node has. The network average degree node is an average over the degree nodes of individuals and equals $\bar{k}=\frac{1}{n} \sum_{i=1}^{n} k_{i}$ where $n$ represents the number of nodes in the network. Very much related to the node degree is the concept of the prestige of individuals. A prestige reflects the number of connections one has in the network (Freeman 1977), whereas it can also refer to the "importance" of connections one has. Using the concept of importance of nodes, Bianconi and Barabasi (2001) define the fitness of each node reflecting the level of attractiveness of a particular node in the network. In the network I use below, the fitness level is considered only implicitly, with all the nodes of the same number of connections having the same level of fitness.

In the simulation games, I assume that all nodes are populated in the network according to the principle of a small world network with individuals having many local and some small number of global connections. The formation of a small world network starts with a regular network where nodes are rewired with some small probability $0<p<<1$. Watts and Strogatz (1998) and Barrat and Weigt (2000) argue that the network has all the properties of a small world network in the neighborhood of $p=0.01$, with the properties changing quite much with the changes in probabilities. In such network, the local homogeneity is large, whereas some global connections drastically reduce the diameter of the network, with the latter being a maximum shortest path between any two nodes in the network.

The last significant characteristic of a network that is being considered here is navigation (Kleinberg 1999). Navigation tackles the question whether individuals are capable of using their connections in such way that they reach their target individual on the network in the shortest possible path. In the paper, I argue that they are not, with individuals tackling the dilemma in such a manner, that in every time interval they choose one from their connections at random. 


\section{The model}

The network is populated with $V=\{1,2, \ldots, 1000\}$ infinitely lived individuals, distributed on the lattice to form a small world network, as defined by Watts and Strogatz (1998). Individuals in the network are rewired with probability $p=0.01$. An average node degree in the network is $\bar{k}=6$. Once individuals are populated on the lattice, the network remains unchanged until the end of the simulation process. A representation of a social network, used in the model is given in Figure 1 with nodes representing individual investors and edges their connections.

Figure 1: Social network of investors

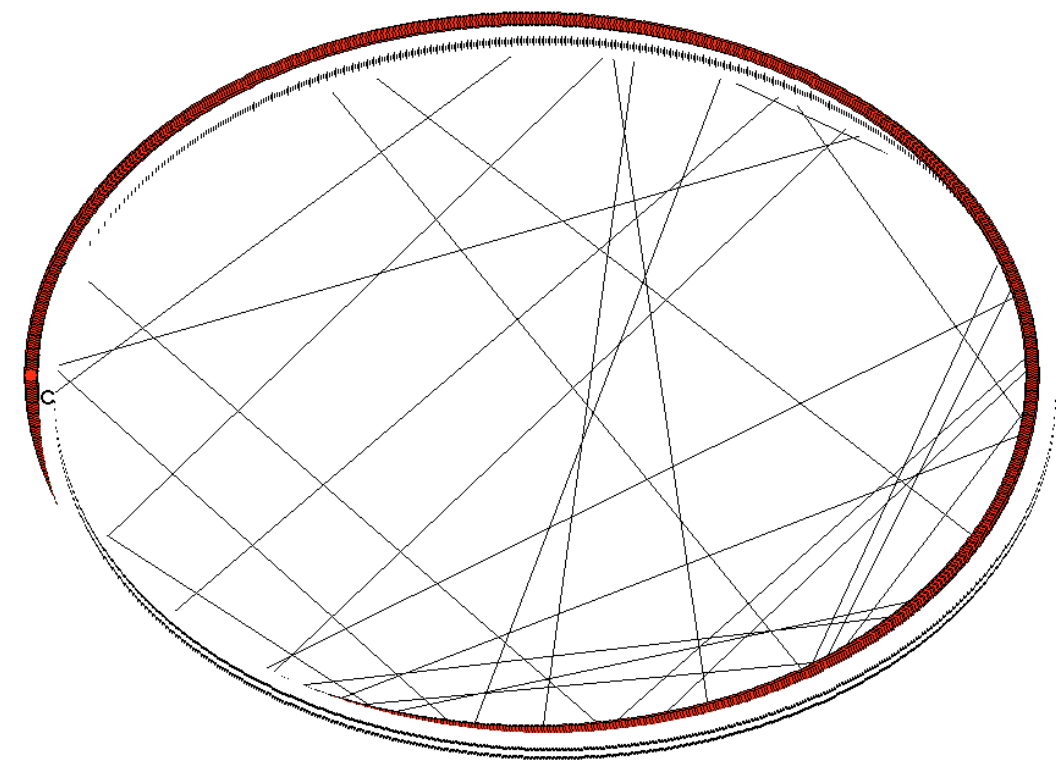

In such network, investors have many local connections and some global. In the model, individuals communicate with others, but make fully autonomous decisions. However, as Levine and Pesendorfer (2007) and Bala and Goyal (1998) argue, the communication within a complete social network leads to synchronous decisions of otherwise autonomous agents.

Individuals accumulate their wealth in time according to the strategy they choose, whereas they are free to change their strategy as the game proceeds. They accumulate the wealth according to (1). 


$$
W_{t+1}\left(A_{(\bullet)}\right)=W_{t}\left(A_{(\bullet)}\right) \cdot\left[r_{t,(\bullet)}\right]
$$

$W_{t+1}\left(A_{(\bullet)}\right)$ and $W_{t}\left(A_{(\bullet)}\right)$ represent the wealth of an individual in time intervals $t$ and $t+1$, while $(\bullet)$ denotes the strategy used by an individual in time, and $r_{t,(\bullet)}$ denotes the return of a strategy $(\bullet)$ in time $t$. Upon their preferences and prospects, individuals can distribute their wealth to all the alternatives that are available to them.

In the game, I use realized returns of stocks on a minute basis. Returns of portfolios are calculated from the returns of individual stocks. Returns of assets are exogenous to them, whereas investors are neither able to foresee them, neither do they know the system that drives returns in time. To make the game feasible, I assume that investors always make portfolio out of equal shares of stocks they include into it. Therefore, if they make portfolio out of three stocks, they have one third of each stock, if they include four stocks into portfolio, they possess one fourth of each stock, and so on.

In the game, I assume that individuals don not make deterministic decisions, but follow a logistic distribution. By doing this, I combine all the kinds of uncertainties of individuals they face when making decisions. This also reflects the level of omniscience of agents. Thus, following Szabo and Toke (1998), the level of omniscience of individuals is defined through $\kappa$.

$$
\wp=\left[1+\exp \left[\left(W\left(A_{i}\right)-W\left(A_{j}\right)\right) / \kappa\right]\right]^{-1}
$$

In every time period $t$ an individual $A_{i}$ chooses one of whom he his directly connected, $A_{j}$, and compares his payoff, $W\left(A_{i}\right)$, to the payoff of selected individual, $W\left(A_{j}\right)$. It depends upon the level of coefficient $\kappa$ which strategy an individual $A_{i}$ adopt. For $\kappa=0$, he always adopts the strategy that gives higher outcome. Such individual is denoted an omniscient individual. Higher the value of $\kappa$ and smaller the difference between the two payoffs, more likely it is that an individual will not choose the strategy with higher payoff. Such individual is denoted a non-omniscient individual. Contrary, lower the value of a coefficient $\kappa$ 
and bigger the difference between the two payoffs, more likely it is that an individual will choose the strategy with higher payoff.

Finally, I assume that a portion of individuals, $l$, never change their strategy, whatever its efficiency. The presence of such liquidity individuals proved to be the most significant for the developments of the game. I set $l=0.1$ and assume that whatever their strategy, these are individuals numbered from $200 \leq n \leq 229,470 \leq n \leq 499,600 \leq n \leq 629$ and $900 \leq n \leq 909$ from $n=1000$ individuals. In the games, liquidity agents pursue all alternatives, randomly defined to them in the beginning of each game.

Figure 2: Pairwise stock returns in time

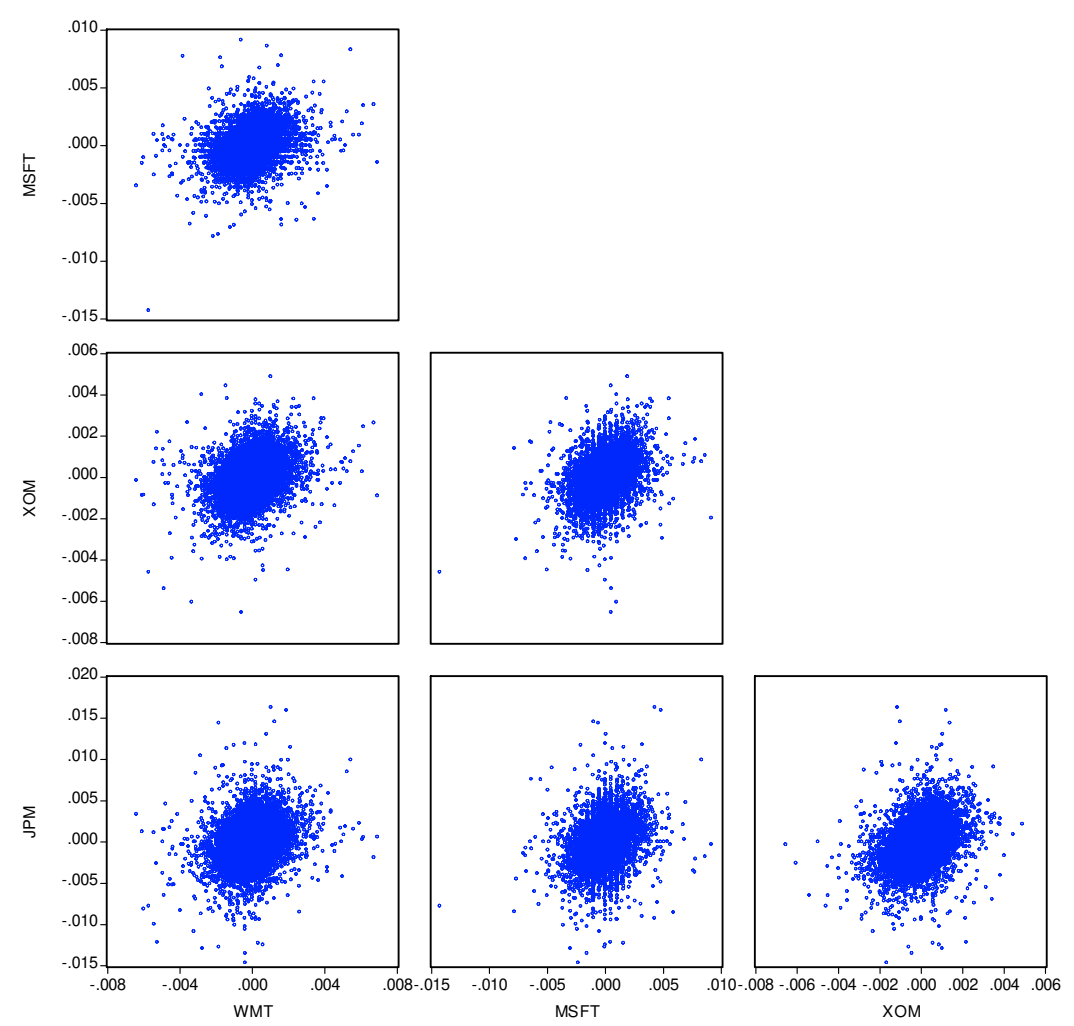

All the games are simulated forward in time using real data of the stocks under consideration. I take the following stocks from the four different sectors: WalMart Stores, Inc. (NYSE: WMT), Microsoft Corporation (NYSE: MSFT), Exxon Mobil Corp. (NYSE: XOM) and JPMorgan \& Chase (NYSE: XOM). The data present the returns of stocks on a minute basis of the trading days' day sessions on the New York Stock Exchange from 9.30 to 16.00. The time period used in the 
simulations is from April 6, 2009 to June 8, 2009. Because of the Memorial Day of May 25, which is a non-trading day, the series refers to 44 trading days and contains 17.160 data for each stock. Pairwise realizations of returns are presented in Figure 2, their distributions in Figure 3 and boxplots in Figure 4.

Figure 3: Distributions of stock returns

Theoretical Quantile-Quantiles
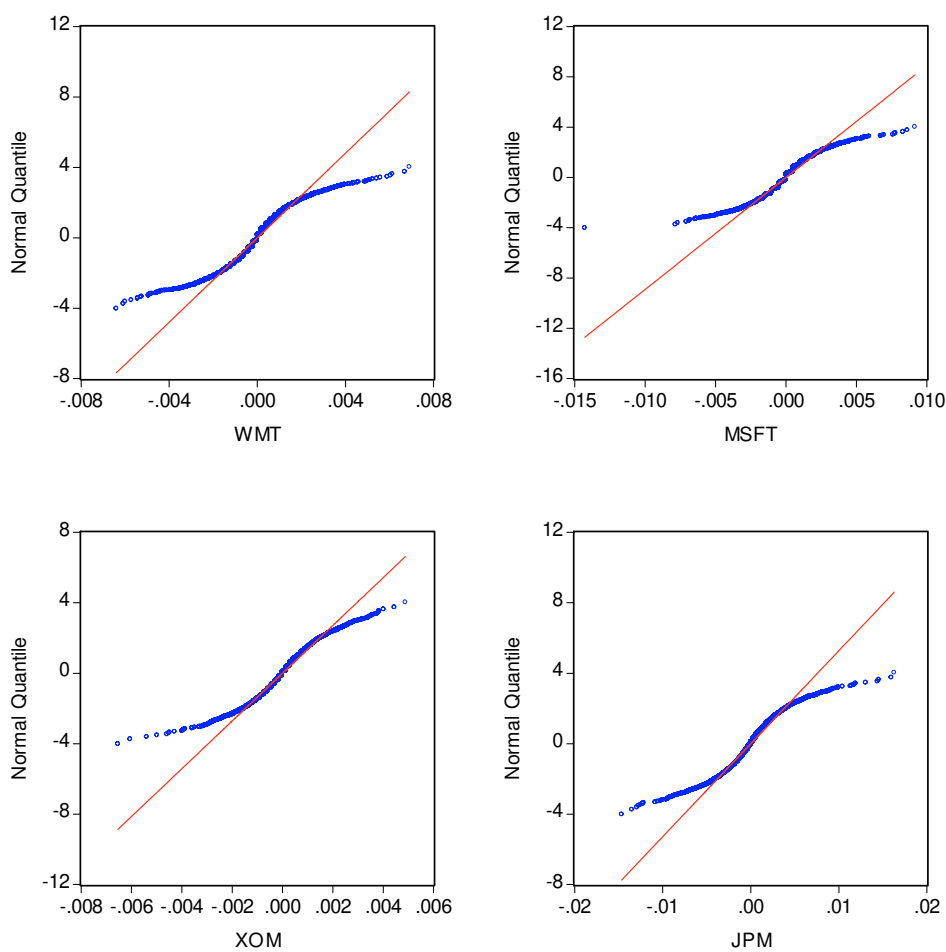

Figure 4: Boxplots of returns

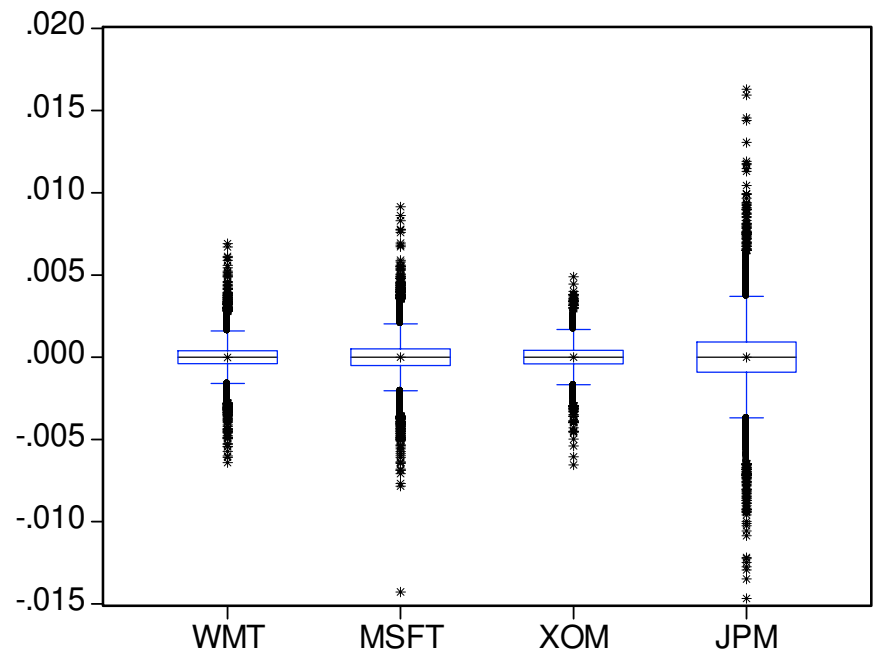


Lower bounds of the boxes in Figure 4 present first quartiles of returns, whereas the upper bounds figure third quartiles of realized returns.

Table 1: Descriptive statistics of returns

\begin{tabular}{lrrrr} 
& \multicolumn{1}{c}{ WMT } & \multicolumn{1}{c}{ MSFT } & \multicolumn{1}{c}{ XOM } & \multicolumn{1}{c}{ JPM } \\
Mean & $-6.51 \mathrm{E}-06$ & $-5.55 \mathrm{E}-06$ & $3.41 \mathrm{E}-06$ & $7.14 \mathrm{E}-06$ \\
Median & 0.000000 & 0.000000 & 0.000000 & 0.000000 \\
Maximum & 0.006905 & 0.009142 & 0.004885 & 0.016282 \\
Minimum & -0.006400 & -0.014271 & -0.006543 & -0.014655 \\
Std. Dev. & 0.000817 & 0.001086 & 0.000734 & 0.001892 \\
Skewness & 0.043424 & -0.026540 & -0.187643 & 0.035034 \\
Kurtosis & 8.880335 & 9.248734 & 6.550736 & 7.940240 \\
& & & & \\
Jarque-Bera & 24728.90 & 27920.39 & 9115.223 & 17453.78 \\
Probability & 0.000000 & 0.000000 & 0.000000 & 0.000000 \\
& & & & \\
Observations & 17160 & 17160 & 17160 & 17160
\end{tabular}

We can see from the Table 1 that all the stocks have excess kurtosis. JPM and MSFT have huge standard deviations, with JPM being very volatile, making it the riskiest of the four. Contrary, the least risky stocks of the four are XOM and WMT.

\section{Simulation results}

\subsection{Omniscient agents}

\section{Two stocks}

I first consider omniscient agents having two different stocks available. They are modeled through the value of the parameter of omniscience $\kappa=0.001$. Because agents can possess either one of the two stocks or equal share of the two, this makes three alternatives available to every investor from which to choose. First, I take WMT and MSFT and simulate two independent realizations of the games. Figures 5 and 6 present the shares of agents of the games playing each of the three alternatives, $s 1, s 2$ and $s 3$, in time. $s 1$ represents the share of agents with WMT, $s 2$ the share of investors with portfolio of the two, and $s 3$ the share of investors with MSFT. 
Figure 5: Shares of agents with each of the alternatives

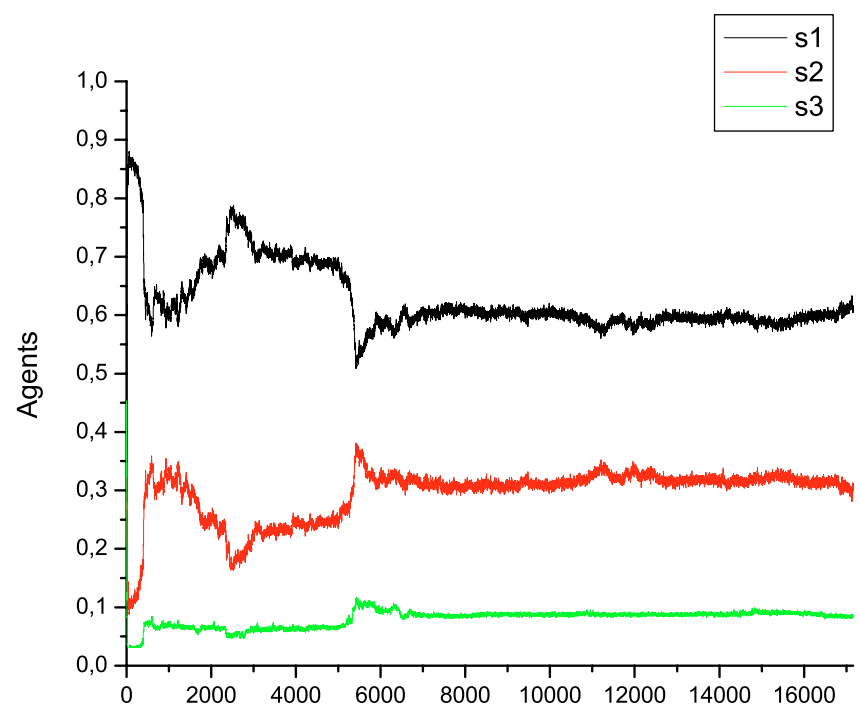

Figure 6: Shares of agents with each of the alternatives

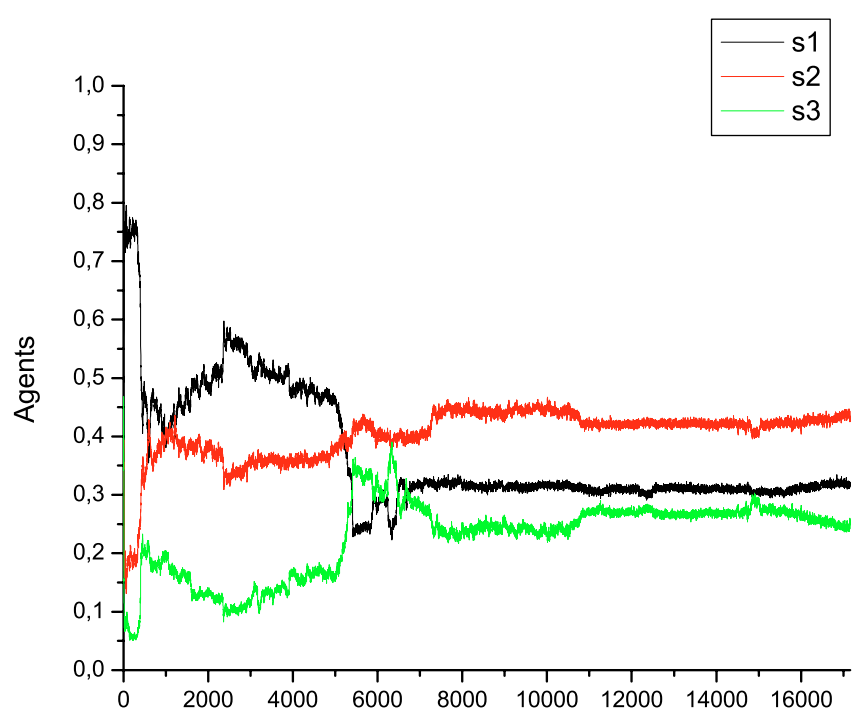

The two figures demonstrate how some consecutive lower performances affect the developments of the game. In such cases, liquidity agents are needed to do their job in "rescuing" dominated alternatives. In the game, they backed MSFT's weak performance from the start, keeping it among the alternatives. We can also detect chaotic behavior of investors from the two figures, as 60.5 percent of investors opt for WMT and 8.3 percent for MSFT in the first realization of the game, whereas 24.8 percent of investors opt for MSFT in the second and 32.2 percent for WMT. The roots of such chaotic behavior lie in the ways in which investors make decisions. That is, an investor first randomly selects an investor to whom he is 
directly connected, compares the two payoffs and makes decision according to (2).

\section{Three stocks}

I now add an additional stock, XOM, into the game, thus increasing the number of alternatives from 3 to 7 . This is so because investors can now choose either to possess only one stock of the three, or equal shares of the two or the three from the three that are available.

Again, I make two independent realizations of the games and present the results in Figures 7 and 8. $s 1$ represents the share of investors with WMT, $s 2$ the share of investors with MSFT, $s 3$ the share of investors with XOM, s4 the share of investors with portfolio of WMT and MSFT, $s 5$ the share of investors with portfolio of WMT and XOM, s6 the share of agents with portfolio of MSFT and $\mathrm{XOM}$, and $s 7$ the share of investors with the portfolio of the three.

Figure 7: Shares of agents with each of the alternatives

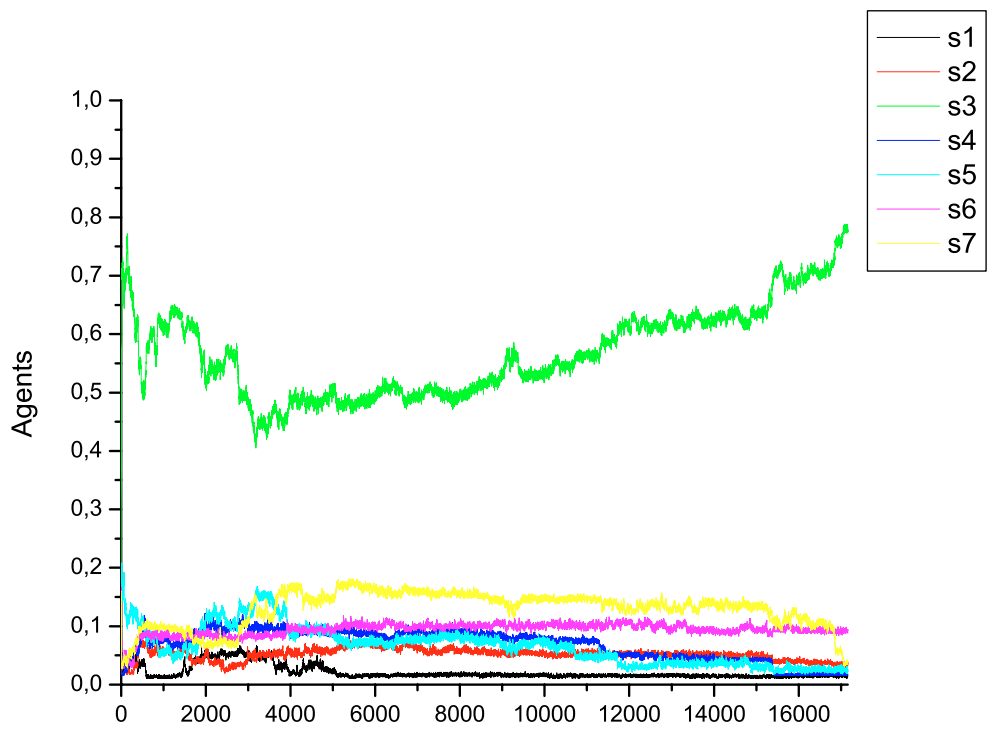


Figure 8: Shares of agents with each of the alternatives

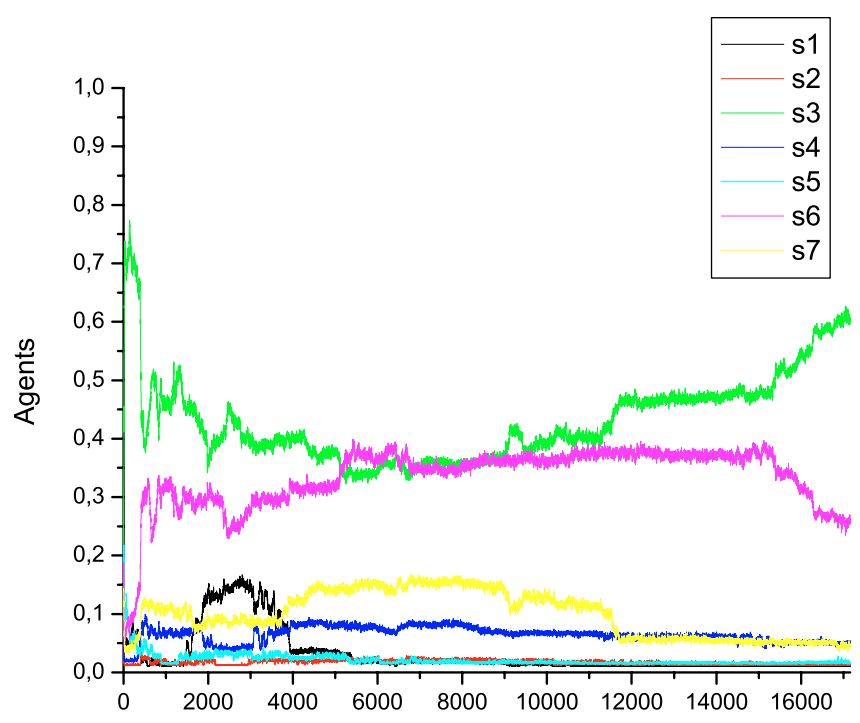

The introduction of XOM, the safest stock among the four, changed the behavior of investors drastically, as XOM turned out to be a dominant and the most desired stock of omniscient investors. Because omniscient investors are very efficient in separating efficient from inefficient alternatives, liquidity agents again take their role of preventing the dominant alternative, this time $\mathrm{XOM}$, to wipe out the others. We can again detect some chaotic behavior in the investors' decisionmaking, with XOM being dominant in the first realization with 78.3 percent of investors choosing it in the last iteration, whereas a portfolio of XOM and MSFT, ended with 25.7 percent, ascended in the second, as MSFT, the riskiest in this setting, improved on the efficiency during the game. XOM ended with 60.3 percent, whereas all the rest ended below 5 percent.

\section{Four stocks}

The last stock I introduce into the game is JPM, which is the riskiest stock of the four. By that, the number of alternatives increases from seven to 15. This is so because investors can now choose either to possess only one of each four stocks, or equal shares of the two, or of the three or of the all four from the four that are present in the game.

Again, I make two independent realizations of the games. Figures 9 and 10 present the shares of agents of the games playing each of the alternatives. $s 1$ represents the share of investors with WMT, $s 2$ the share of investors with 
MSFT, $s 3$ the share of investors with XOM, $s 4$ the share of investors with JPM. $s 5$ represents the share of investors with portfolio of WMT and MSFT, $s 6$ the share of agents with portfolio of WMT and XOM, $s 7$ the share of investors with the portfolio WMT and JPM, $s 8$ the share of investors with the portfolio MSFT and XOM, s9 the share of investors with the portfolio MSFT and JPM, s10 the share of investors with the portfolio XOM and JPM. s11 represents the share of investors with the portfolio WMT, MSFT and XOM, s12 the share of investors with the portfolio WMT, MSFT and JPM, s13 the share of investors with the portfolio WMT, XOM and JPM, s14 the share of investors with the portfolio MSFT, XOM and JPM, and $s 15$ the share of investors with the portfolio of the all four.

Figure 9: Shares of agents with each of the alternatives

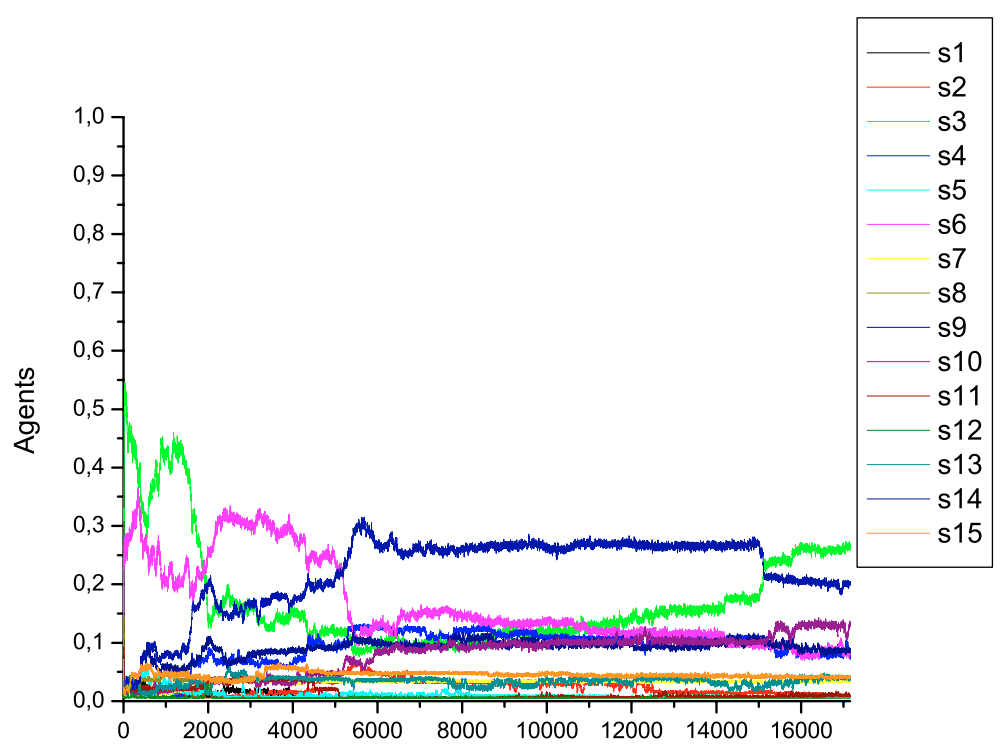


Figure 10: Shares of agents with each of the alternatives

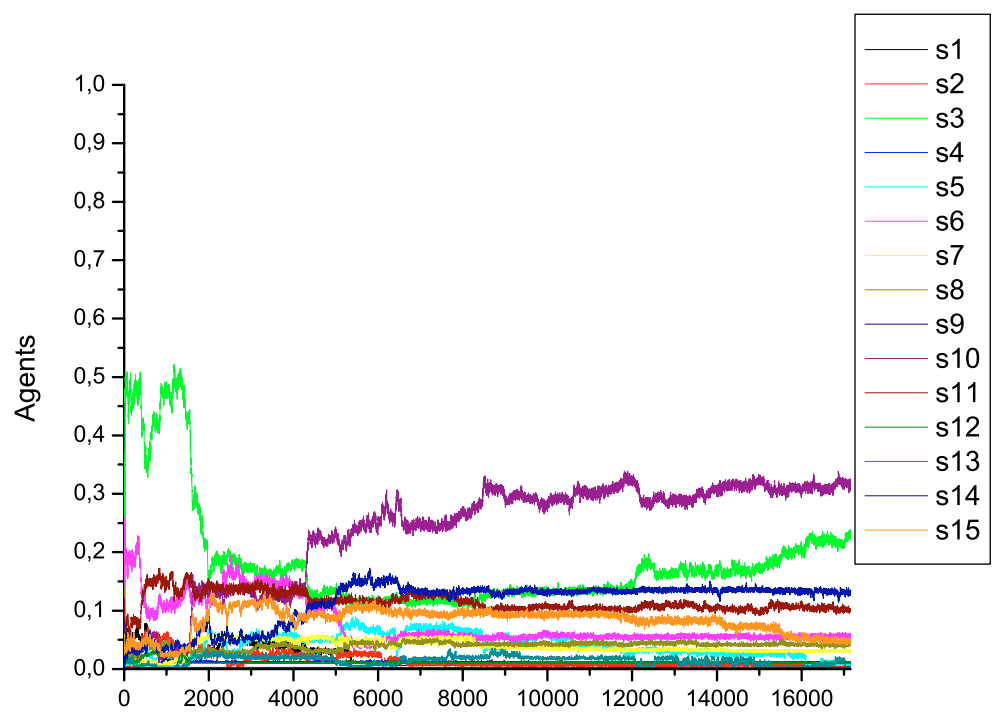

After the introduction of JPM, the riskiest of the four, XOM ended first in the first realization of the game with 26.6 percent of investors opted for it, second being portfolio MSFT, XOM and JPM with 20.4 percent. 12.6 percent of investors opted for portfolio of the least and the most risky stock, XOM and JPM, with 8.9 percent for the portfolio of the two riskiest, JPM and MSFT. That is slightly higher then the share of investors opting for the portfolio of the two safest stocks, XOM and WMT (8.1 percent), or only for the JPM (7.9 percent).

In the second realization of the game, portfolio of XOM and JPM ended first with 31 percent, followed by XOM with 23.3 percent, portfolio of portfolio MSFT, XOM and JPM with 13.2 percent and portfolio of WMT, MSFT and XOM with 10.1 percent. We can find some similarities when comparing the two. In both realizations XOM, and portfolios of XOM and JPM, and MSFT, XOM and JPM were among the most desired in the end. We might conclude from such behavior that investors back their risk with some safest stocks, but also that they are prepared to take the risk.

\section{More than four stocks}

Using the assumptions of the game, having more than four stocks in the game increases the number of alternatives sharply. Namely, they equal to the number of combinations that could be made out of the $n$ stocks available in the game, i.e. 
$C(n, i)=\sum_{i=1}^{n}\left(\frac{n !}{i !(n-i) !}\right)$ with $i$ representing the number of stocks from all the stocks in the game that an investor includes into his portfolio. Therefore, with five stocks in the game an investor has 31 different alternatives available, with six stocks there are 63 alternatives, with seven stocks 127, with 15 stocks 32.767 alternatives, etc.

When knowing that DJIA is made out of 30 stocks and that there are 500 stocks in the Standard\&Poors, whereas the NASDAQ composite index includes more than 3.800 stocks, we see that there are a way too many alternatives available than investors could handle.

\subsection{Non-omniscient agents}

Finally, I take non-omniscient agents and do the same groups of simulations again. To model non-omniscient agents, I take $\kappa=0.5$. Color palette and the alternatives used in figures equals to that in the omniscient agents.

\section{Two stocks}

When deciding from the two different stocks, each agent has three alternatives available from which to choose. Again, I take WMT and MSFT and make two independent realizations of the games. Figures 11 and 12 present the shares of agents of the games playing each of the alternatives available. 
Figure 11: Shares of agents with each of the alternatives

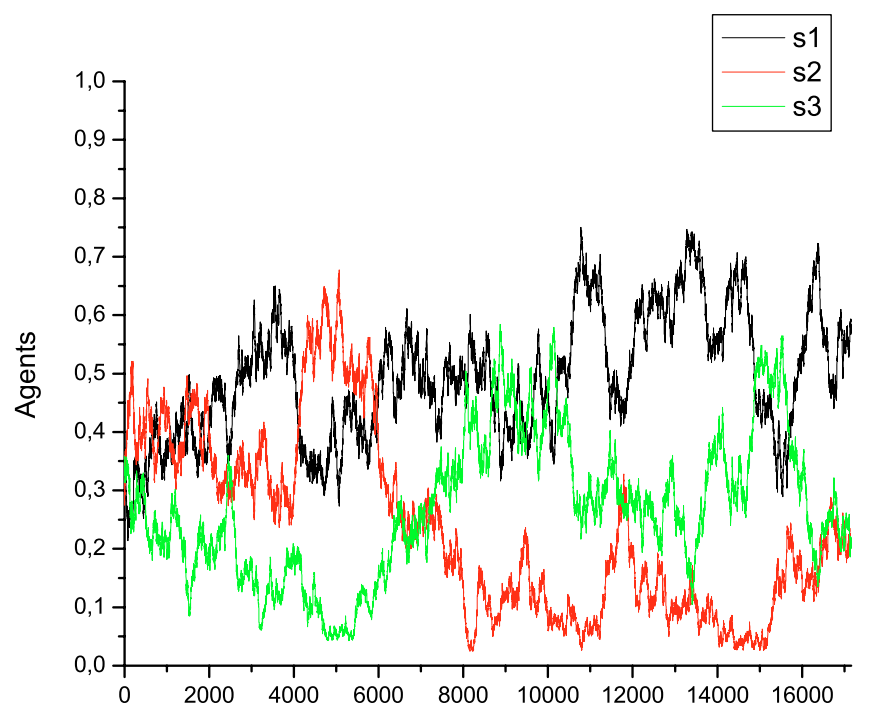

Figure 12: Shares of agents with each of the alternatives

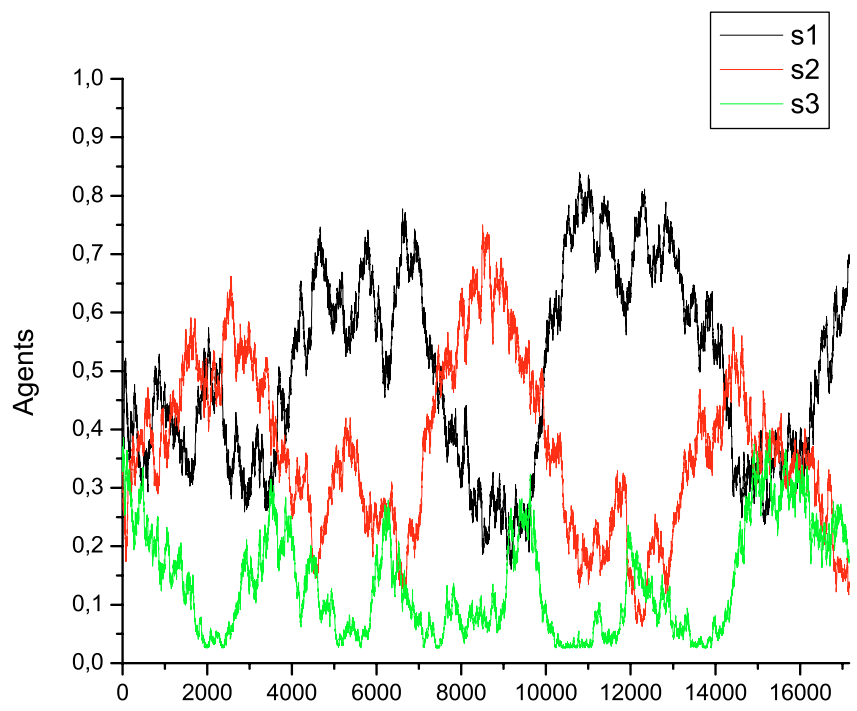

The introduction of non-omniscient investors drastically changed the behavior of investors, which became very much changeable in time, similar to the chaotic. In both cases, investors value WMT a better alternative in the end, with 58.4 percent in the first realization and 70 percent in the second. The other alternatives were valued pretty much the same.

\section{Three stocks}

As in the case of omniscient agents, I introduce a XOM stock into the game, thus increasing the number of alternatives to seven. I make two independent 
realizations of the games and present the results in Figures 13 and 14. Figures present the shares of agents of the games playing each of the alternatives available to them.

Figure 13: Shares of agents with each of the alternatives

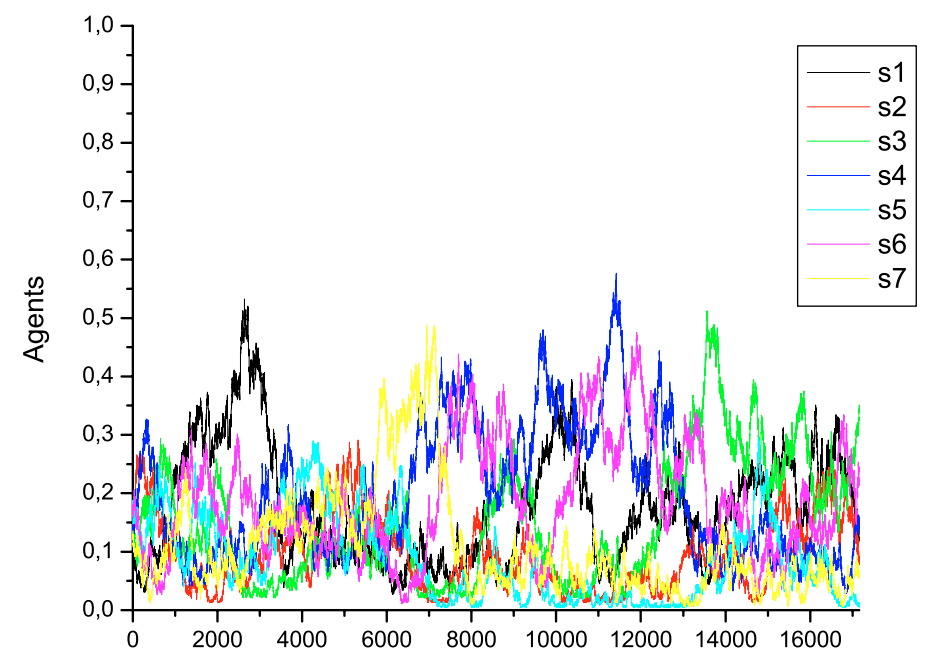

Figure 14: Shares of agents with each of the alternatives

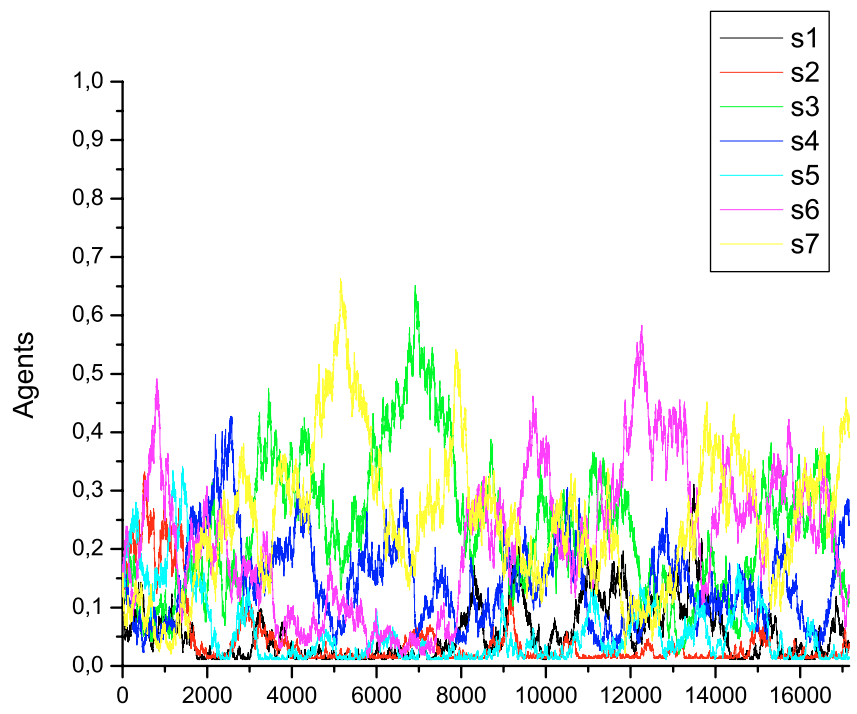

With three stocks and seven alternatives available, non-omniscient investors make their decisions very arbitrary. In the last iteration of the first realization of the game, 33.8 percent of them opted for XOM, and 21.7 percent for portfolio of MSFT and XOM. The order of precedence is the same as in a game of omniscient investors' setting, but shares here are fundamentally different. In the second realization of the game, most investors (40 percent) opted for portfolio of all the 
three, being followed by portfolio of WMT and MSFT (28.4 percent), and portfolio of MSFT and XOM with 12.6 percent. In the first realization, a winning portfolio of the three was opted by 6.1 percent of investors, and was backed by liquidity agents. When comparing also other alternatives, we see that nonomniscient investors decide randomly.

\section{Four stocks}

Finally, I include JPM and increase the number of alternatives to 15 . The results of two independent realizations of the games are presented in Figures 15 and 16.

Figure 15: Shares of agents with each of the alternatives

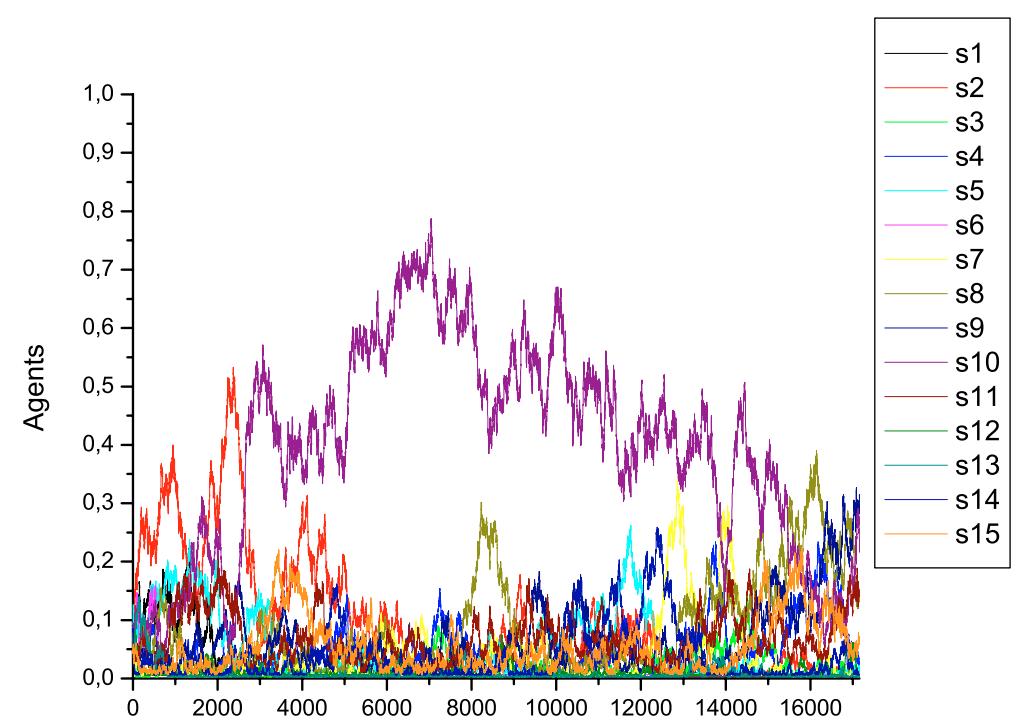


Figure 16: Shares of agents with each of the alternatives

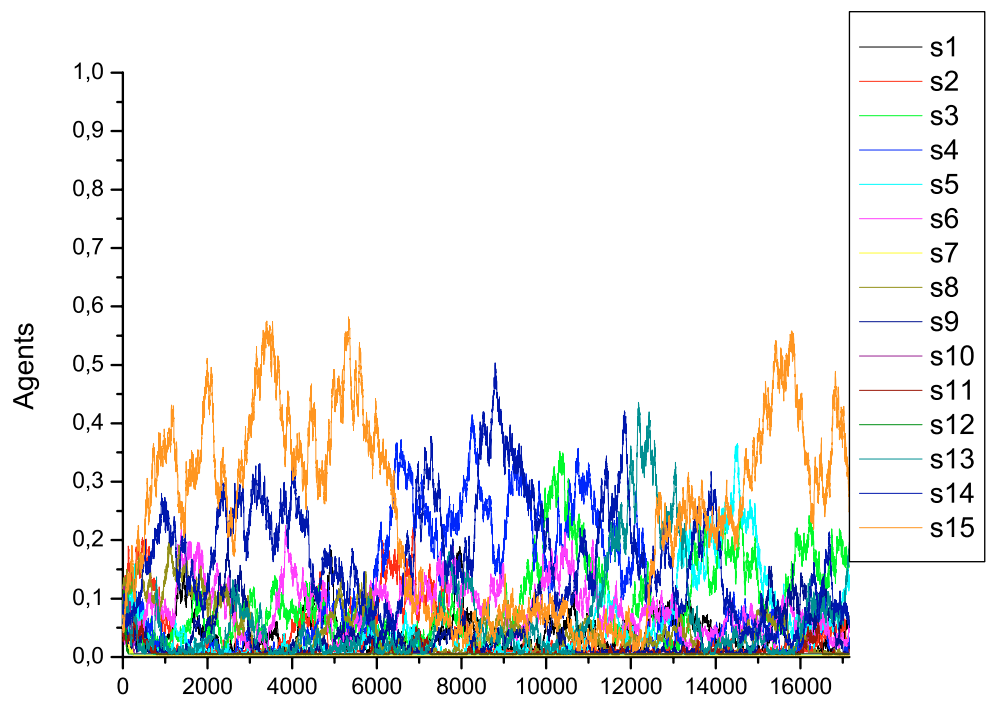

The winning alternative of the first realization of the game was a portfolio of MSFT and JPM with 31 percent investors opting for it, being followed by a portfolio of XOM and JPM with 21.6 percent and portfolio of WMT, MSFT and $\mathrm{XOM}$ with 15.2 percent. In the second realization of the game, the winning alternative was the portfolio of the all four stocks with 25.9 percent, being followed by portfolio of WMT, XOM and JPM with 19.2 percent and XOM with 18.6 percent. When comparing the two realizations, the winning portfolio of all the four stocks was chosen by 6.4 percent of investors in the first realization of the game. Contrary, the winning alternative of the first game, portfolio of MSFT and JPM, was chosen by only 1.6 percent of investors in the second game, and the second best in the second game, portfolio of WMT, XOM and JPM, was chosen by 0.3 percent of investors in the first realization of the game, thus ended on the liquidity investors. There are no common patterns when combining the two realizations of the games and figures indicate that non-omniscient investors make decisions chaotically.

\section{Concluding remarks}

Simulations put some answers on how individual investors make their portfolios when they have many alternatives available. It has been demonstrated that most of the time omniscient investors took the safest stock, or combine it with the riskiest. Therefore, to improve on the efficiency and securing the riskiness of the 
alternative, omniscient investors usually take portfolio of one risky stock, either JPM or MSFT, and back the risk with the one of the safest, usually with XOM. Contrary, the behavior of non-omniscient investors pretty much reflects that of the chaos.

Liquidity agents have again proved to be indispensable elements in the games. However, as the number of alternatives increases, the probability that they fail in performing their role of keeping all the non-dominant alternatives alive also increases. Namely, it happens that some of them are caught among the others, which means that they share their information only with other liquidity agents, who do not change their strategies. As this happens, investors who do change alternatives in time lose information about individual alternatives, making such alternatives to fall beyond their reach.

Finally, managing portfolio is very complex and tough task to do, especially when there are too many alternatives that are changing in time available. Therefore, also omniscient investors behave in a chaotic manner, making the prediction of their behavior harder. Besides, neither are they able to reach a unanimous decision in time, as returns of alternatives change much faster then information are able to circle through the entire network.

\section{REFERENCES}

1. Bala, Venkatesh, and Sanjeev Goyal (1998). "Learning from Neighbours." Review of Economic Studies 65(3): 595-621.

2. Barrat, Alain, and Martin Weigt (2000). "On the Properties of Small-World Networks.” European Physics Journal B 13(3): 547-560.

3. Becker, Gary (1996). Accounting for Tastes. Harvard: Harvard University Press.

4. Bianconi, Ginestra, and Albert-Laszlo Barabasi (2001). "Competition and Multiscaling in Evolving Networks.” Europhysics Letters 54(4): 436-442.

5. Bikhchandani, Sushil, David Hirshleifer, and Ivo Welch (1998). "Learning from the Behavior of Others: Conformity, Fads, and Information Cascades." Journal of Economic Perspectives 12(3): 151-170. 
6. Campbell, John, Andrew Lo, and Craig MacKinlay (1997). The Econometrics of Financial Markets. Princeton: Princeton University Press.

7. Fama, Eugene (1965). "The Behavior of Stock Prices." Journal of Business 38(1): 34-105.

8. Freeman, Linton (1977). "A Set of Measures of Centrality Based on Betweenness." Sociometry 40(1): 35-41.

9. Hayek, Friedrich August von (1945). "The Use of Knowledge in Society." American Economic Review 35(4): 519-530.

10. Hirshleifer, David (2001). "Investor Psychology and Asset Pricing." Journal of Finance 56(4): 1533-1597.

11. Kahneman, Daniel, and Amos Tversky (1979). "Prospect Theory: An Analysis of Decision under Risk." Econometrica 47(2): 263-291.

12. Keynes, John Maynard (1936). General Theory of Employment, Interest, and Money. Cambridge: Macmillan.

13. Levine, David, and Wolfgang Pesendorfer (2007). "The Evolution of Cooperation through Imitation." Games and Economic Behavior 58(2): 293315.

14. Markowitz, Harry (1952). "Portfolio Selection.” Journal of Finance 7(1): 7791.

15. Osborne, Martin, and Ariel Rubinstein (1990). Bargaining and Markets. New York: Academic Press.

16. Rubinstein, Ariel (1998). Modeling Bounded Rationality. Cambridge: MIT Press.

17. Russel, Stuart, and Peter Norvig (1995). Artificial Intelligence: A Modern Approach. New Jersey: Prentice Hall.

18. Steinbacher, Matjaz (2008a). "Evolutionary Portfolios through the Small World Network.” FSI Working Paper 5. http://ssrn.com/abstract=1160412.

19. Steinbacher, Matjaz (2008b). "Knowledge, Preferences, and Shocks in a Portfolio Analysis." Working Paper. http://ssrn.com/abstract=1317319.

20. Steinbacher, Matjaz (2009a). "Acceptable Risk in a Portfolio Analysis." Working Paper. http://ssrn.com/abstract=1320993.

21. Steinbacher, Matjaz (2009b). "The Role of Liquidity Individuals in the Decision-Making.” Working Paper. http://ssrn.com/abstract=1336151. 
22. Steinbacher, Matjaz (2009c). "What is the 'Value' of Value-at-Risk in a Simulated Portfolio Decision-Making Game?" Working Paper. http://ssrn.com/abstract=1355529.

23. Szabo, Gyorgy, and Csaba Toke (1998). “Evolutionary Prisoner's Dilemma Game on a Square Lattice." Physical Review E 58(1): 69-73.

24. Wasserman, Stanley, and Katherine Faust (1998). Social Network Analysis: Methods and Applications. Cambridge: Cambridge University Press.

25. Watts, Duncan, and Steven Strogatz (1998). "Collective Dynamics of Small World Networks." Nature 393(4): 440-442. 